\title{
The Finite-Precision Numerical Effects Analysis of Infinite Impulse Response
}

\author{
Ganbaatar Nandintsetseg, Xiang-E Sun \\ School of Electronic \& Information, Yangtze University, Jingzhou, China \\ Email: nandia0627@gmail.com
}

How to cite this paper: Nandintsetseg, G. and Sun, X.-E. (2019) The Finite-Precision Numerical Effects Analysis of Infinite Impulse Response. Open Access Library Journal, 5: e4719.

https://doi.org/10.4236/oalib.1104719

Received: June 12, 2018

Accepted: January 7, 2019

Published: January 10, 2019

Copyright $\odot 2019$ by authors and Open Access Library Inc.

This work is licensed under the Creative Commons Attribution International License (CC BY 4.0).

http://creativecommons.org/licenses/by/4.0/

(c) (i) Open Access

\begin{abstract}
This paper mainly uses the MATLAB software to design IIR digital filter with the amplitude frequency characteristic method and zero-pole method, and then analyzes the effect of coefficient quantization and quantization effects in IIR digital filter arithmetic. It is that we can use the software to draw some pictures. These pictures can show coefficient system function of IIR digital filter with a certain length to quantify the zero pole after the system distribution, frequency response, after quantizing the coefficients under the direct, parallel and cascade structures, as well as the limit cycle oscillation and the overflow oscillation of fixed-point operation in the quantization effect of operation are drawn.
\end{abstract}

\section{Subject Areas}

Computer Engineering, Multimedia/Signal Processing

\section{Keywords}

Infinite-Impulse Response (IIR), Filter Design, Word Length, Error,

Graphical User Interface (GUI)

\section{Introduction}

In theoretical analysis of discrete-time systems, we generally assume that signal values and system coefficients are represented in the real-number system. However, with analog discrete-time systems, the limited precision of the components of a circuit makes it difficult to realize coefficients exactly. Similarly, when implementing digital signal processing systems, we must represent signals and coefficients in some digital number that must always be of finite precision [1]. Most general-purpose digital computers, DSP chips, or special-purpose hardware use a binary system. The coefficients of digital filters designed by theory 
method are infinite in precision. And all filter coefficients must be in binary form of limited length stored in memory, so we must quantify the ideal value. It will bring error between the actual coefficient and original coefficient, and cause the deviation of zero pole position, thus affecting the performance of the filter. If the coefficients quantization error is large, pole of IIR digital filter may be removed from the unit circle, making the system unstable and not available. Effect of digital filter coefficient quantization is related to word length, as well as the filter structure [2] [3]. The analysis of sensitivity of filter pole can reflect the influence of coefficient quantization having on zero and pole position in different filter structures.

In addition, calculation will also bring error. The finite word length effect in the computation process has a complex relationship with the system used (fixed-point, floating-point system), the code system (the original code, complement) and quantization (rounding, truncating) [3].

\section{MATLAB Implementation the Effects of Quantization on the Performance in IIR Filters}

Based on the analysis of the effects of learning quantization on the performance of IIR filters, we designed and implemented a MATLAB-based quantization effect analysis system for the performance of IIR digital filters. Using this system, the IIR system can be analyzed different word length conditions as shown Figure 1.

\subsection{Overall System Framework Design in Matlab}

The quantization effect of IIR digital filters [4] [5] is mainly manifested in two aspects:

1) The Operational quantization effect.

The basic mathematical operation for implementing a digital filter is a constant that multiplies some number or two numbers. Where there is a multiplication of coefficients, there is a source of quantization error noise, which can all cause errors in the output of the coefficients.

2) The Coefficient quantization effect.

The effect of coefficient quantization on the filter is related to the word length, and it is also closely related to the structure of the filter. Analysis of the zero-pole sensitivity can reflect the effect of coefficient quantification on the position of the zero pole in the case of different structures. After analysis, it can be concluded that the deviation of the pole formed by the sensitivity of the pole position cannot directly obtain the deviation of the frequency response. Especially in the high-order case, there are many coefficients, and their quantization errors are more random. Therefore, statistical analysis method is used to consider the coefficient quantization error as a random variable, so as to analyze the deviation of the estimated frequency response of the filter.

\subsection{Matlab GUI Design}

The GUI designer is a software application used for the design. Upper part of the panel provides menu buttons and common tools, such as the left offers a variety 


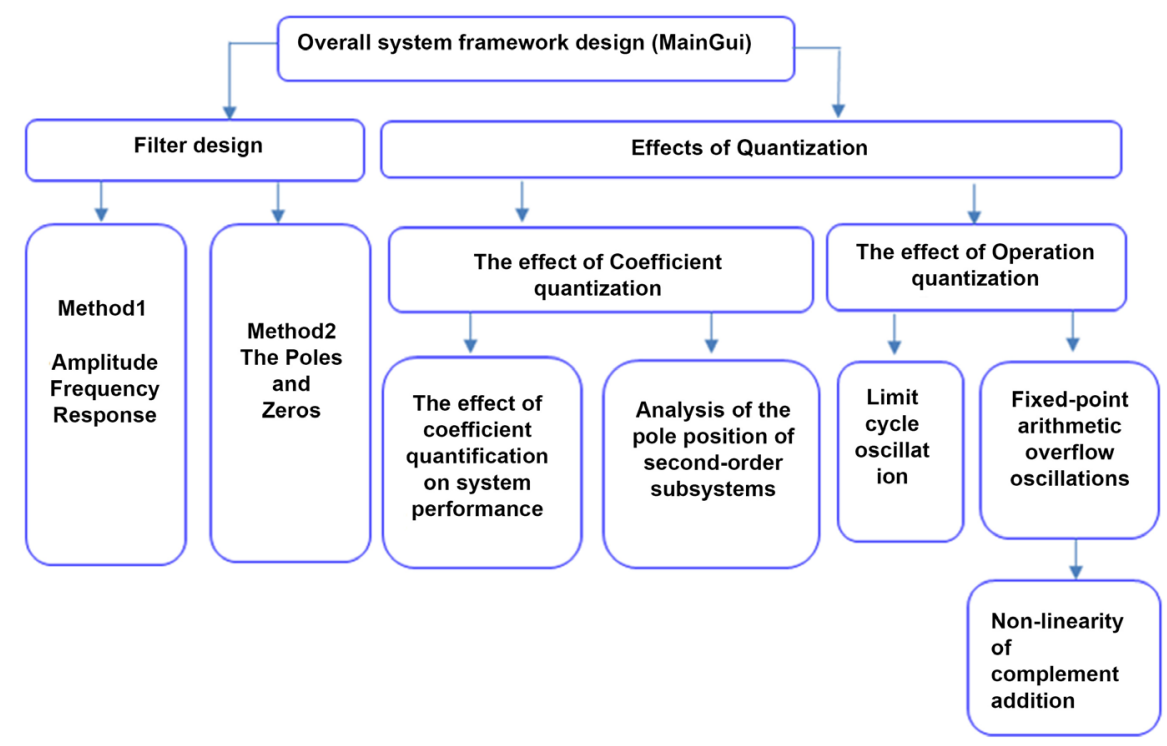

Figure 1. Overall system diagram.

of command buttons, radio buttons, editable text box, static text boxes, pop-up menus etc.

Therefore, when setting these properties [5], pay attention to the following common and important property settings:

1) Control style and appearance

Background Color: set the background color of the control, use [R G B] or color definition.

Foreground Color: text color.

String property: the text on the control, as well as the options of the list box and popup menu.

2) The general information of the object

Enable property: Indicates the enable state of this control. It is set to on.

Style: Control object type.

Tag: control representation (user-defined).

User Data: User specified data.

Font attributes such as Font Angle, Font Name, etc.

3) The implementation of the control callback function

Busy Action: The interrupt handling the callback function.

ButtonDownFcn property: The processing function when the button is pressed.

CallBack attribute: It is the link of the substantive function of the entire program system of the connection program interface.

The current status of the control

4) Listbox Top: The index of the top, most string displayed in the list box.

Max/Min: The maximum or minimum value.

Value: The current value of the control.

5) It is very convenient to use MATLAB to make such a process, we can see 
through the GUI operation. Generally should complete the following two steps such as Figure 2:

a) GUI interface design. Mainly through the use of many tools such as different text boxes, buttons, etc. Graphical user interface (GUI) to understand what the function of this graphical interface is, that is the operation on the graphical interface. What kind of result will result from doing?

b) The design of the callback function. The user should aim at different drawings according to the function of the designed graphical interface. Shape object to write the function code that can achieve this function, to ensure that this graphical interface can be complete Scheduled features.

\section{The Effect of Coefficient Quantization Analysis in IIR Digital Filters}

\subsection{Amplitude-Frequency Indicators IIR Filter Design Method}

In this example, choose the IIR Cheby I low-pass filter, bandpass frequency wp = $0.4 \pi$, stopband frequency ws $=0.6 \pi$, bandpass filters maximum attenuation $\mathrm{rp}=$ $1 \mathrm{DB}$, stopband filters minimum attenuation $\mathrm{rs}=40 \mathrm{DB}$, sampling frequency fs $=1 \mathrm{~Hz}$. Filters are designed with these parameters.

Click result button the amplitude-frequency and phase-frequency filters responses are drawn curve like Figure 3. This method can be used to design high-order filters, and the effects of quantization effects on the zero-pole of the filter system can be clearly observed [6] [7].

\subsubsection{Statistical Analysis of Frequency Response Deviation}

The amplitude-frequency characteristics of the quantized coefficients of the digital filter deviate from the original amplitude-frequency characteristics, and this deviation is related to the quantized word length.

$$
H(z)=\frac{\sum_{i=0}^{M} b_{i} z^{-i}}{1-\sum_{i=1}^{N} a_{i} z^{-i}}=\frac{B(z)}{A(z)}, \hat{H}(z)=\frac{\sum_{i=0}^{M} \hat{b}_{i} z^{-i}}{1-\sum_{i=1}^{N} \hat{a}_{i} z^{-i}}
$$
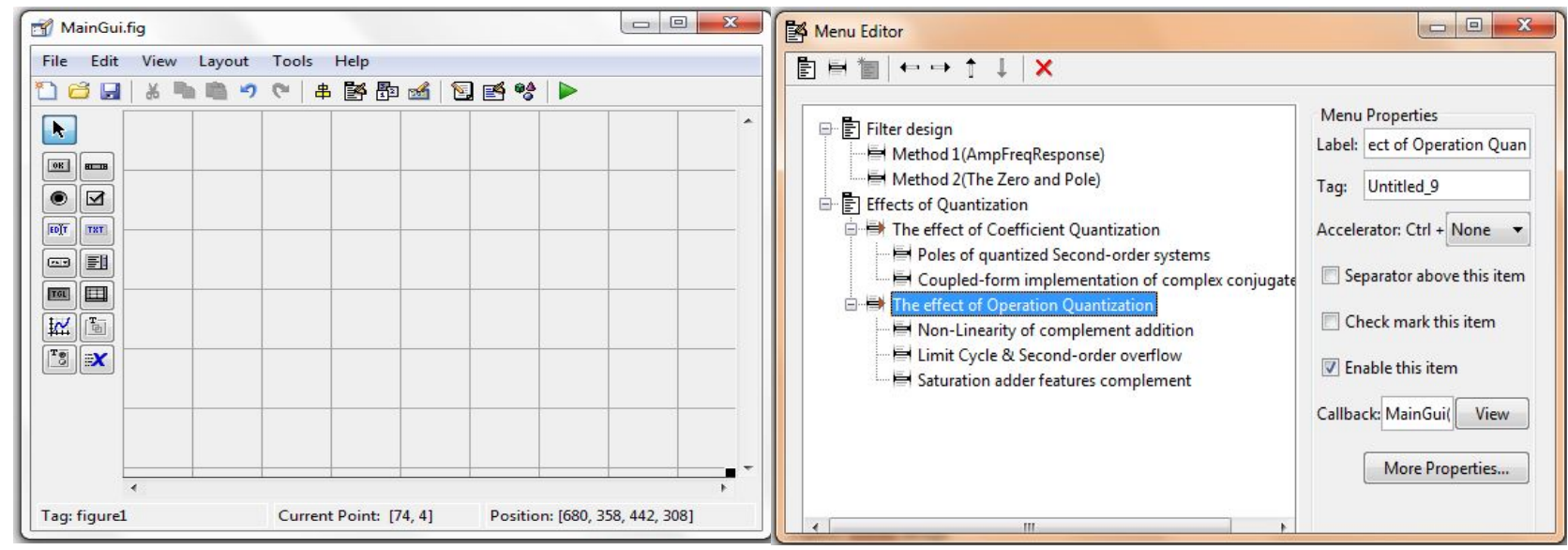

Figure 2. Matlab design. 


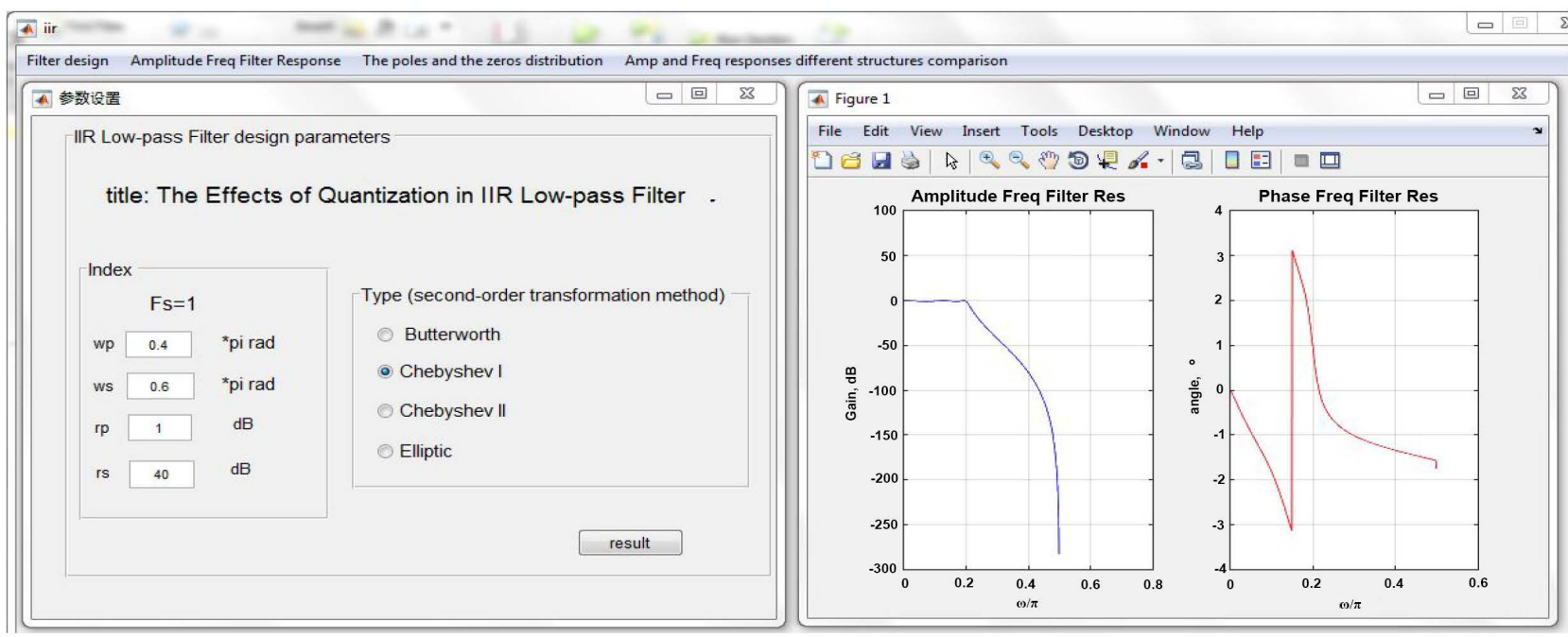

Figure 3. Designed amplitude \& phase frequency response.

From Figure 3, amplitude \& phase frequency response is already designed after the coefficients are quantized by 4 bits, the corresponding amplitude-frequency response has been seriously distorted, its attenuation characteristics have been degraded, and the distortion of the amplitude-frequency response after 8-bit quantization has been significantly reduced. After the 32-bit quantization, the amplitude-frequency response has approached the original amplitude-frequency response. Figure 4 can be seen that the quantization of the coefficients will distort the amplitude-frequency response, and the method of reducing the errors is to increase the quantized word length.

\subsubsection{Quantization Effect Analysis of Different Structures}

Three different structures can be used to implement an IIR filter: direct, cascade, and parallel type. The filter coefficients are quantized, and the deviation of the amplitude and frequency characteristics of the processed filters from the original amplitude-frequency characteristics is also closely related to the implementation structure. Through programming, draw a comparison chart of the amplitude-frequency response when different structures are implemented, and distinguish them with different colors.

As can be seen from Figure 5, whether it is 4 bit quantization or 8 bit quantization, the effect of cascade and parallel implementation is far better than the direct implementation. At the same time, the parallel implementation is slightly better than the cascade implementation. Parallel implementation is the smallest error.

\subsection{The Zero-Pole Method}

The IIR digital filter meter filter is designed based on the parameters of the zero point $\mathrm{Z}$, the pole $\mathrm{P}$ and the gain $\mathrm{A}$, and the quantization effect of the filter is analyzed. With the zero-pole method, the user can set the zero-pole position and the gain of the system to facilitate changing the system pole-zero. 


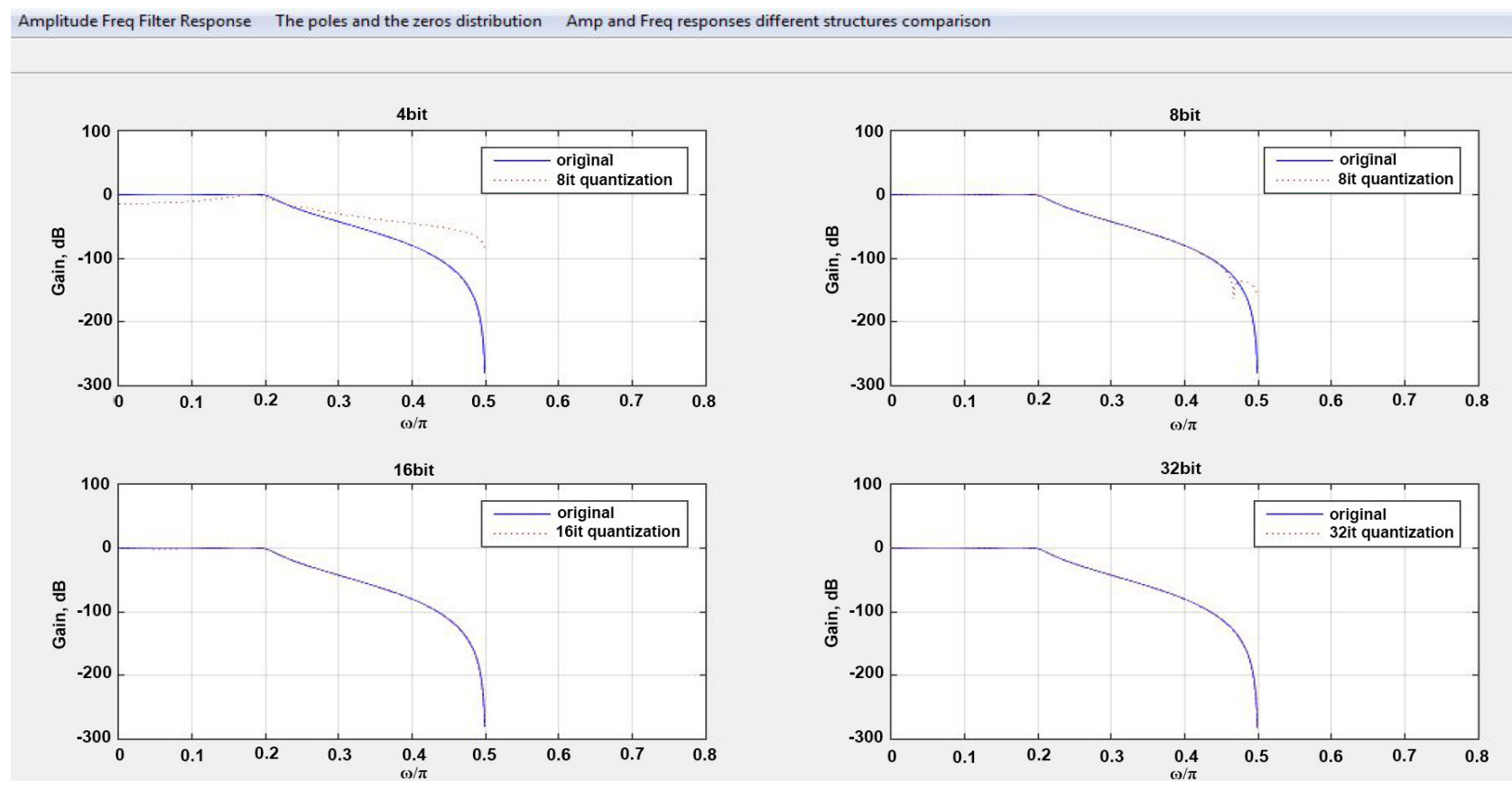

Figure 4. Different word lengths after quantization.


Figure 5. Frequency response of different structures.

The amplitude-frequency and phase-frequency filters responses are drawn curve. The zero-pole method can obtain the transfer function of the filter through the zero point, pole, and system gain, and the design is relatively simple. Zero-pole and system gain indicators can be directly changed in Figure 6. 


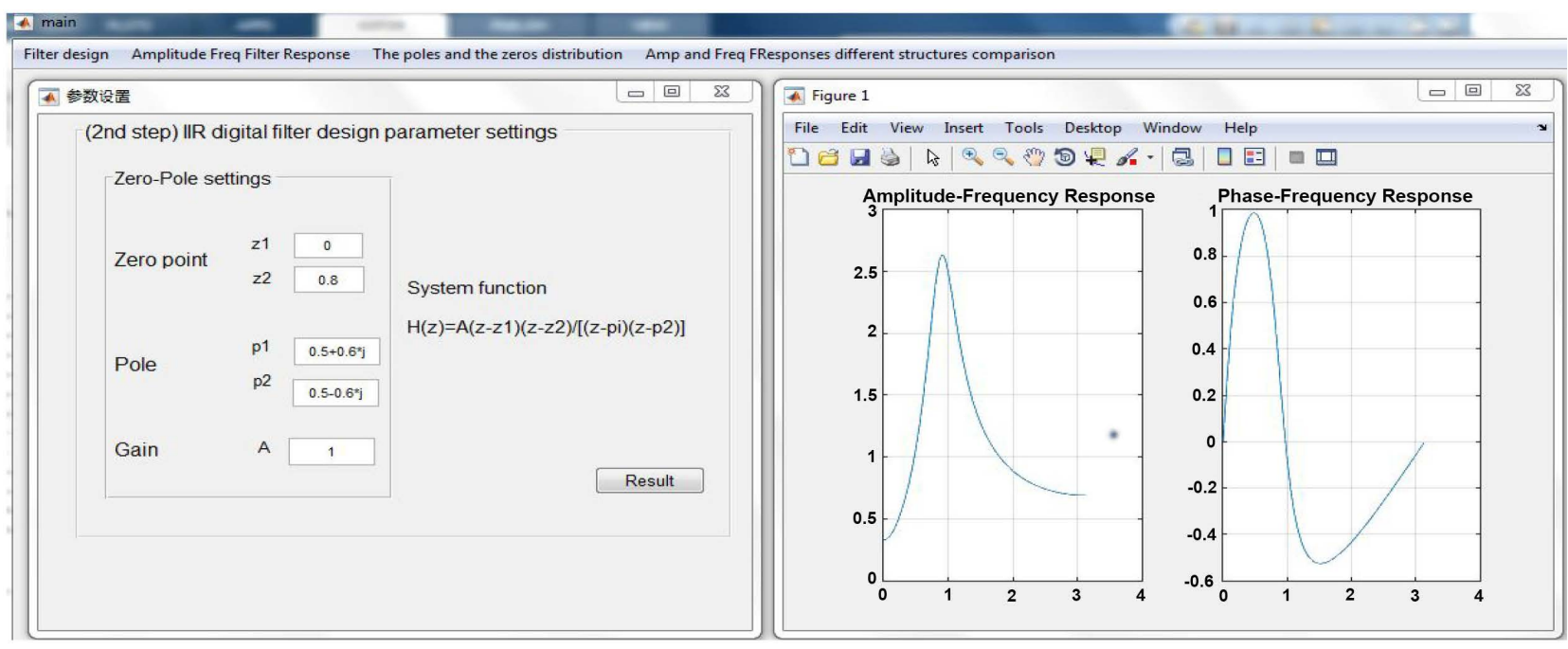

Figure 6. The zero-pole filter design interface.

However, this method is not suitable for designing high-order digital systems. Designing low-order systems is not a significant effect of observing quantization effects on the pole-zero of IIR filters.

\subsubsection{The Effects of Coefficient Quantization Influence on the Zero-Pole Method}

The stability of the filter depends on the position of the pole. If the quantization error of the coefficient causes the pole within the unit circle to move to or away from the unit circle, the characteristic of the filter is different from the required frequency response, and the stability of the filter is affected.

For example, the band-pass filter with conjugate poles near the imaginary axis is shown Figure 7(a), and the low-pass filter with conjugate poles near the real axis is shown Figure 7(b).

As a result of the destruction, it is clear that this is most likely to occur in the poles closest to the unit circle within the unit circle. Comparing the four plots in Figure 8, we can see that the poles of the original system are all within the unit garden.

After the 4 bit quantization, some poles go beyond the unit circle, and the system becomes stable and unstable. After 8 bit quantization, the system is stable. After the coefficients of the IIR digital filter are quantized, the poles may go out of the unit circle, making the system unstable, increasing the quantized word length, improving stability, and reducing errors.

\subsubsection{Poles of Quantized Second-Order Systems}

The cascade or parallel forms are based on second-order modules. Despite the simple structure of a second-order system, there are still several ways to implement it such that the quantization effects on the pole locations can be diminished. Consider a second order system $H(z)=\frac{1}{1+a_{1} z^{-1}+a_{2} z^{-2}}$ therefore the coefficients $r^{2}=a_{2}, \quad r \cos \theta=-\frac{a_{1}}{2}$ will be quantized [8] [9] [10]. 




(a)



(b)

Figure 7. The pole position response is inversely proportional to the distance between poles. (a) Long distance from poles; (b) Short distance from poles.
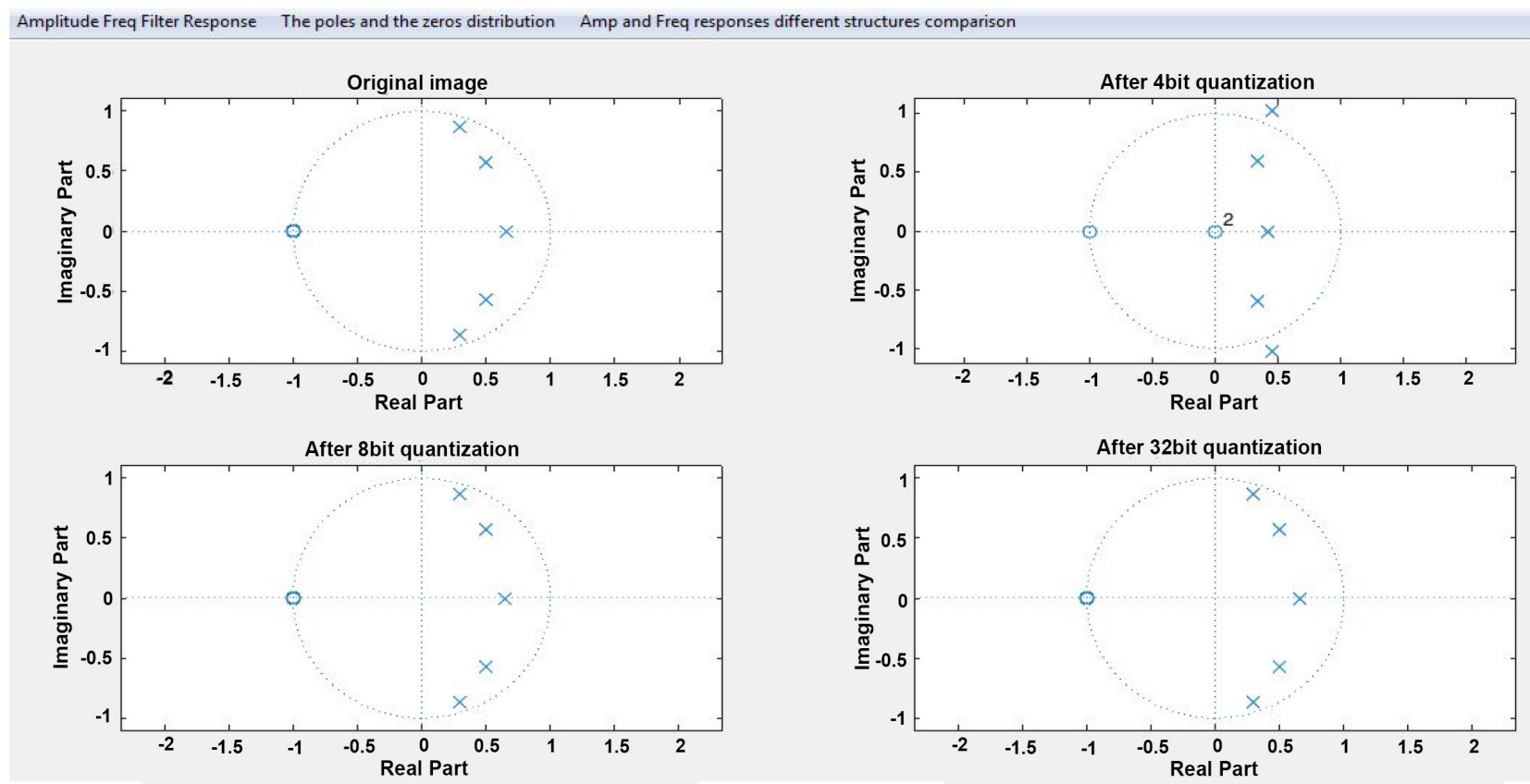

Figure 8. Original and Quantified zero-pole diagram.

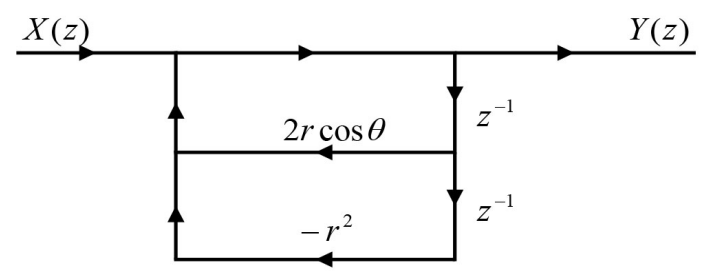

Figure 9. Direct-form implementation of a complex-conjugate pole pair.

Notice that for the direct form, the grid is rather sparse around the real axis.

The plots of Figure 10 are, of course, symmetrically mirrored into each of the other quadrants of the z-plane. Thus poles located around $\theta=0$ or $\theta=\pi$ maybe shifted more than those around $\theta=\pi / 2$. It is always possible that the infinite-precision pole location is very close to one of the allowed quantized poles. 


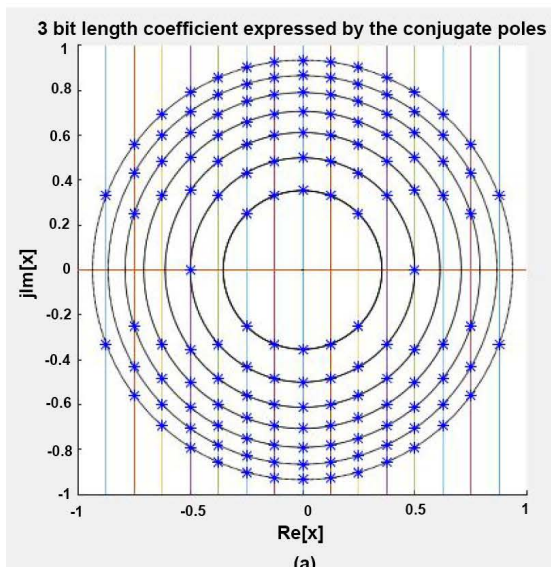

(a)

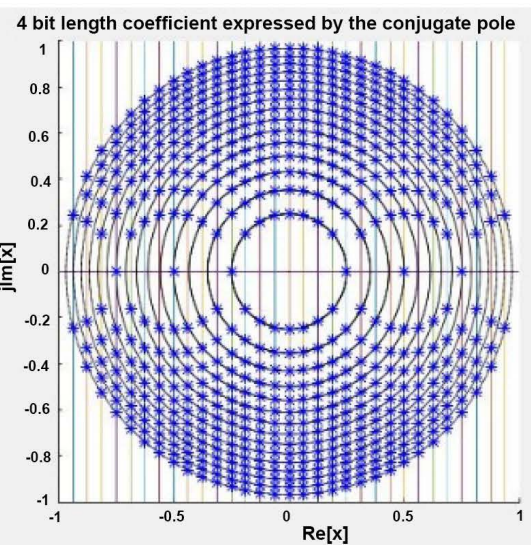

(b)

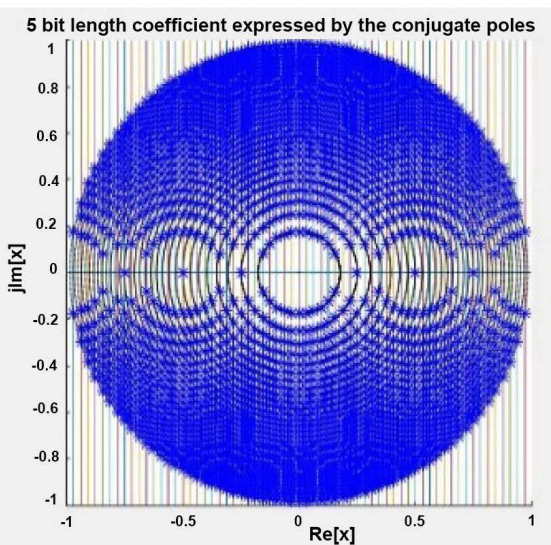

(c)

Figure 10. Pole locations for the second-order IIR direct-form system of Figure 9. (a) Three-bit quantization (b) Four-bit quantization (c) Five-bit quantization.

\subsubsection{Coupled-Form Implementation of Complex-Conjugate Pair}

An alternative second-order structure for realizing poles at $z=r e^{j \theta}$ and $z=r e^{-j \theta}$ is shown in Figure 11. This structure is referred to as the coupled form for the second-order system.

It is easily verified that the systems of Figure 10 and Figure 12 have the same poles for infinite-precision coefficients. Since these quantities are the real and imaginary parts, respectively, of the pole locations, the quantized pole locations are at intersections of evenly spaced horizontal and vertical lines in the z-plane. Figure 12(a)-(c) show the possible pole locations for 3-bit to 5-bit quantization, respectively. In this case, the density of pole locations is uniform throughout the interior of the unit circle. Twice as many constant multipliers are required to achieve this more uniform density. In some situations, the extra computation might be justified to achieve more accurate pole location with reduced word length.

\section{Conclusions}

The quantization effect affects the performance of the IIR digital filter, which changes the zero-pole position of the filter system. After the quantization, the system pole may go beyond the unit cell, which makes the system unstable. After the quantification, the amplitude-frequency response of the system may be severely distorted, and the attenuation characteristics of the system may be degraded.

By completing the design content, the basic principle of the IIR digital filter and the effect of the quantization effect on the performance of the IIR digital filter are summarized. The possible errors in the implementation of IIR system are analyzed, and the solution to the problem is proposed. The statistical analysis of the IIR digital filter's coefficient quantization effect, operation and quantization effect, and fixed-point complement overflow-limit ring oscillation phenomenon and low-level limit-ring oscillation caused by fixed-point rounding are analyzed to explain the cause of the limited word length effect, the impact of errors, and 




(a)

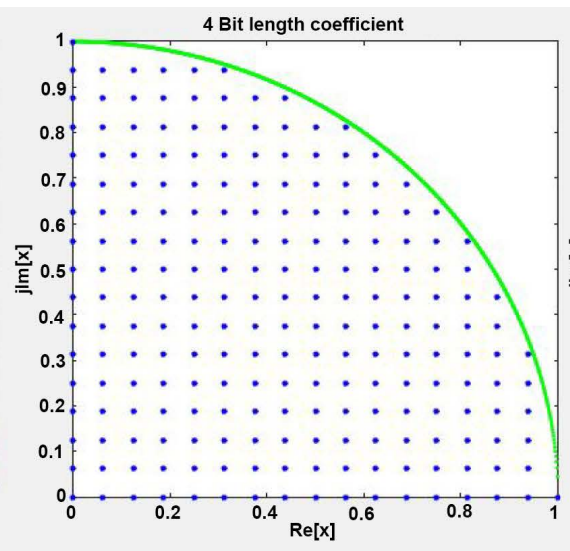

(b)

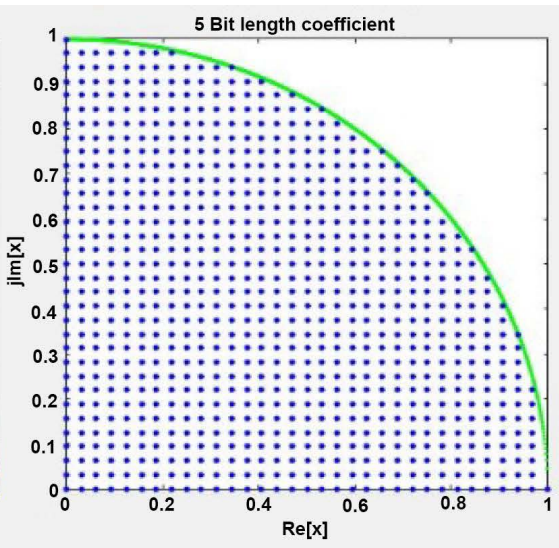

(c)

Figure 12. Pole locations for coupled-form second-order IIR system of Figure 11. (a) Three-bit quantization (b) Four-bit quantization (c) Five-bit quantization.

the effect of the structure on stability. In this paper, the digital filter is designed by using the amplitude-frequency characteristic method and zero-pole method of MATLAB programming, and the coefficient quantization effect and the operation and quantization effect of the digital filter are analyzed.

That is to say, the zero-pole distribution and amplitude-frequency response of the system after quantizing the coefficients of the system function of IIR digital filter with a certain word length, and the frequency response of the system after quantizing the coefficients under the direct, parallel and cascade structures, as well as the related figures of the operational quantization effect are drawn. The effect of coefficient quantification on the position of zeros and poles is closely related to the distribution of zero and pole positions and the structure of the filter. Increasing the quantized word length allows the system pole to be close to the unit circle, improving the frequency response characteristics.

The direct type structure has the largest output error; the cascade type has the second; and the parallel type has the smallest error. For the IIR filter, from the limited word length effect, no matter which type of direct type structure is the worst, the operation error is the greatest, and it should be avoided when it is used in high-order. The cascaded structure is better, and the parallel structure 
has the smallest operational error.

To reduce the effect of finite word length, it should:

1) increase the word length appropriately;

2) select the appropriate filter structure;

3) as far as possible, avoid direct-type structures for systems above the second-order system, and use parallel-type and cascade-type structures.

\section{Conflicts of Interest}

The authors declare no conflicts of interest regarding the publication of this paper.

\section{References}

[1] Oppenheim, A.V., Schafer, R.W. and Buck, J.R. (1998) Discrete-Time Signal Processing. 2nd Edition, Prentice-Hall, Inc., Upper Saddle River, New Jersey.

[2] Lyons, R.G. (2011) Understanding Digital Signal Processing. 3rd Edition, Upper Saddle River, New Jersey.

[3] Zhang, Z. (2013) Analysis of Quantization Effects on the Performance of the IIR Digital Filter. Journal of School of Electronics \& Information, No. 6, 25-45.

[4] Ingle, V.K. and Proakis, J.G. (2008) Digital Signal Processing and Matlab Implementation. 2nd Edition, Publishing House of Electronics Industry, Beijing.

[5] Chapman, S.J. (2008) MATLAB ${ }^{\circledast}$ Programming for Engineers. 2nd \& 4th Edition, CL Engineering, CA, USA.

[6] Smith, S.W. (1999) The Scientist and Engineer's Guide to Digital Signal Processing. 2nd Edition, CL Engineering, CA, USA.

[7] Parhi, K.K. (2007) VLSI Digital Signal Processing Systems: Design and Implementation. John Wiley \& Sons, California Technical Publishing.

[8] Hsiao, T. (2014) Digital Signal Processing Lecture 15, Finite-Precision Numerical Effects. National Chiao Tung University.

[9] Singh, R. and Arya, K. (2012) Genetic Algorithm for the Design of Optimal IIR Digital Filters. Department of Electronics and Communication Engineering, GJUS\&T, Hisar, India.

[10] Burrus, S.S. (1993) Computer-Based Exercises for Signal Processing Using MATLAB. Prentice Hall PTR, NJ, USA. 